\title{
Receptor tyrosine kinase expression in high-grade gliomas before and after chemoradiotherapy
}

\author{
KUANYU WANG ${ }^{1,2^{*}}$, RUOYU HUANG ${ }^{2 *}$, CHENXING WU ${ }^{3 *}$, GUANZHANG LI $^{2}$, \\ ZHENG ZHAO ${ }^{4}$, HUIMIN $\mathrm{HU}^{4}$ and YANWEI LIU ${ }^{5}$

\begin{abstract}
${ }^{1}$ Department of Gamma Knife Center, Beijing Tiantan Hospital, Capital Medical University, Beijing 100070;
${ }^{2}$ Department of Neurosurgery, Beijing Neurosurgical Institute; ${ }^{3}$ Department of Neurosurgery, Sanbo Brain Hospital, Capital Medical University; ${ }^{4}$ Department of Neuropathology, Beijing Neurosurgical Institute; ${ }^{5}$ Department of Radiotherapy, Beijing Tiantan Hospital, Capital Medical University, Beijing 100050, P.R. China
\end{abstract}

Received February 24, 2018; Accepted March 28, 2019

DOI: $10.3892 / \mathrm{ol} .2019 .11017$

\begin{abstract}
Glioma is the most common type of malignant brain tumor, and is characterized by invasive growth and chemoradiotherapy resistance. The following Cancer Genome Atlas mutation subtypes were identified in initial high-grade gliomas and recurrent gliomas treated by chemoradiotherapy: Isocitrate dehydrogenase 1/2 (IDH1/2) mutation, epidermal growth factor receptor variant III (EGFRvIII) mutation, tumor protein P53 mutation, PTEN mutation, $\mathrm{O}^{6}$-methylguanine-DNA methyltransferase promoter methylation and telomerase reverse transcriptase (TERT) mutation. The expression profile of 58 receptor tyrosine kinases (RTKs) were also examined. It was revealed that the proneural tumor subtype and IDH1/2 mutation are more frequent in recurrent tumors compared with initial tumors. Lower frequencies of the classical subtype, EGFRvIII mutation and TERT mutation were identified in recurrent tumors. A set of six RTK genes in which the level of expression was influenced by chemoradiotherapy was identified. Survival analysis revealed that the expression of several RTKs, including apoptosis-associated tyrosine kinase, fibroblast growth factor receptor 1 and insulin-like growth factor 1 receptor (IGF1R), was associated with patient survival. The stimulation of glioma cells by IGF1 in vitro was found to decreased the viability of the cells following treatment with temozolomide (TMZ). In addition, the expression level of IGF1R was increased in glioma cells treated with TMZ. These data suggest that altered RTK expression levels may influence the sensitivity of glioma to chemoradiotherapy.
\end{abstract}

Correspondence to: Dr Yanwei Liu, Department of Radiotherapy, Beijing Tiantan Hospital, Capital Medical University, 6 Tiantan Xili, Dongcheng, Beijing 100050, P.R. China

E-mail: liuyanwei_tiantan@163.com

*Contributed equally

Key words: glioma, glioblastoma, receptor tyrosine kinases, chemoradiotherapy, biomarker

\section{Introduction}

Malignant gliomas, particularly glioblastomas (GBMs), are the most common primary tumors of the central nervous system (1). According to World Health Organization (WHO) classification, malignant gliomas mainly include WHO grade II, grade III and GBM (WHO-grade IV) (2). High-grade glioma, including grade III and GBM, is the most malignant type and has the worst prognosis, requiring significant lesion resection combined with radiotherapy or/and temozolomide (TMZ) chemotherapy (3). Patients with GBM have a mean survival time of only 14.4 months (1), which is likely due to chemoradiotherapy resistance. Therefore, new therapeutic targets that increase sensitivity to chemoradiotherapy are urgently needed. Evidence suggests that therapeutic effects can be altered by gene alterations. Gliomas exhibiting isocitrate dehydrogenase 1/2 (IDH1/2) mutations are associated with a relatively good prognosis compared with wild-type (4). Studies have found that the expression of mutant IDH1 causes the glioma hypermethylator phenotype, which may serve a critical role in glioma treatment $(5,6)$. Oligodendrogliomas exhibiting loss of chromosome $1 \mathrm{p} / 19 \mathrm{q}$ respond favorably to combined procarbazine, lomustine and vincristine treatment (7-9). Researchers have demonstrated that the loss of chromosome $1 \mathrm{p} / 19 \mathrm{q}$ is associated with the downregulation of tumor suppressor genes or the mutation of specific genes, including CIC and NOTCH1 $(10,11) . \mathrm{O}^{6}$-methylguanine-DNA methyltransferase (MGMT) promoter methylation has been used to guide glioblastoma treatment decisions (12). Resistance to TMZ therapy is thought to arise when the methylated DNA bases are repaired by MGMT, or when the mismatch repair (MMR) pathway functions deficiently (12). Patients with high-grade glioma exhibiting promoter hypermethylation of the MGMT gene, which silences its expression, have been shown to respond well to TMZ therapy $(1,12)$. These results suggest that gene alterations serve important roles in the regulation of chemoradiotherapy sensitivity in gliomas.

Receptor tyrosine kinases (RTKs), a family of cell-surface receptors, constitute important signaling pathways in tumor development (13). RTKs are key regulators of critical cellular processes, including proliferation and differentiation, 
cell survival, metabolism and migration, and cell cycle control (13-15). In the human genome, a total of 58 RTKs have been identified $(16,17)$. All RTKs have an extracellular ligand-binding domain, a single transmembrane helix and a cytoplasmic region that has protein tyrosine kinase activity (13). Agonist binding to the extracellular domain evokes dimerization, and leads to autophosphorylation of the tyrosine kinase domain in a 'trans' orientation, which serves as a site of assembly of protein complexes and stimulates multiple signaling pathways $(13,16)$. RTKs are of widespread interest as drug targets in numerous types of cancer, including epidermal growth factor receptor (EGFR) in metastatic non-small-cell lung cancer and vascular endothelial growth factor in metastatic colorectal carcinoma (14). A number of diseases, including glioma and lung cancer, result from genetic changes or abnormalities that alter the activity, abundance, cellular distribution and/or regulation of RTKs $(10,17)$. It has been reported that alterations of RTKs, including mutation, expression changes or rearrangement can result in drug resistance. EGFR mutation serves a critical role in resistance to gefitinib in lung cancer with anaplastic lymphoma kinase gene rearrangement (18). It has also been found that RTK activation may increase sensitivity to chemotherapy. High levels of hepatocyte receptor tyrosine kinase (HGFR/Met) signaling pathway activation by hepatocyte growth factor (HGF) can increase sensitivity to the Met inhibitor crizotinib in patients with glioma (19). Therefore, it is hypothesized that the expression of RTKs may influence TMZ chemotherapy in patients with high-grade glioma.

In the present study, gene alterations before and after chemoradiotherapy were compared in terms of various Cancer Genome Atlas subtypes, including IDH1/2 mutation, EGFR variant III (EGFRvIII) mutation, tumor protein P53 (TP53) mutation, phosphatase and tensin homolog (PTEN) mutation, MGMT promoter methylation and telomerase reverse transcriptase (TERT) mutation, as well as the expression profile of 58 RTKs. The results demonstrated that, when stimulated by growth factors, the activation of insulin-like growth factor 1 receptor (IGF1R) signaling increased tumor cell sensitivity to TMZ, and TMZ was able to influence IGF1R expression. Survival analysis demonstrated that high expression of IGF1R predicts longer overall survival in patients treated with TMZ.

\section{Materials and methods}

Data collection. The characteristics of patients were collected from the Chinese Glioma Genome Atlas (CGGA; http://www.cgga.org.cn). Samples that were used for RNA sequencing, classical marker detection and survival analysis were from patients who underwent surgical resection between January 2005 and December 2015. The information about radiotherapy and chemotherapy treatment in patients with recurrent glioma was as follows: 22 patients received radiotherapy and 27 patients received chemotherapy. Patients were eligible for the study if their diagnosis was established histologically by two neuropathologists, according to the 2007 WHO classification guidelines (2). Only samples composed of $\geq 80 \%$ tumor cells were selected for analysis. The total number of patients included in the analysis was 182 . This study was approved by the Ethics Committee of Beijing Tiantan Hospital
(Beijing, China), and written informed consent was obtained from each patient.

Cell lines. The human GBM cell lines, $\mathrm{H} 4$ and LN-229, were obtained from the Institute of Biochemistry and Cell Biology, Chinese Academy of Sciences (Shanghai, China). The H4 cell line is derived from an astrocytoma patient who was originally diagnosed with a neuroglioma, and the LN-229 cell line is derived from a patient with glioblastoma (https://www.atcc.org/). Cells were maintained in DMEM (Gibco; Thermo Fisher Scientific, Inc.) containing 10\% fetal bovine serum (FBS; Gibco; Thermo Fisher Scientific, Inc.), $50 \mathrm{U} / \mathrm{ml}$ penicillin $\mathrm{G}$ and $250 \mu \mathrm{g} / \mathrm{ml}$ streptomycin in a humidified atmosphere containing $5 \% \mathrm{CO}_{2}$ at $37^{\circ} \mathrm{C}(20)$. Cells with or without stimulation for $30 \mathrm{~min}$ with fibroblast growth factor (FGF; PeproTech; $10 \mathrm{ng} / \mathrm{ml}$ ) or IGF1 (PeproTech, $100 \mathrm{ng} / \mathrm{ml}$ ) were then treated with $\mathrm{TMZ}$ at $37^{\circ} \mathrm{C}$ for $1-5$ days $(50 \mu \mathrm{M}$; Sigma-Aldrich; Merck KGaA) $(21,22)$.

Western blot analysis. Whole-cell lysates were prepared using RIPA buffer (Cell Signaling Technology, Inc.). The protein determination used Pierce Bicinchoninic Acid Protein Assay kit (Thermo Fisher Scientific, Inc.). Equal amounts of total protein $(30 \mu \mathrm{g})$ were separated by $10 \%$ SDS/PAGE, transferred to a PVDF membrane (EMD Millipore) and detected using an ECL Western Blotting Detection System (Bio-Rad Laboratories, Inc.). 5\% skim milk was used to block the membrane at room temperature for $1 \mathrm{~h}$. Primary rabbit polyclonal antibodies against IGF1R (ProteinTech Group, Inc.; cat. no. 10297-1-AP; dilution, 1:200) were used at $4^{\circ} \mathrm{C}$ overnight. An antibody against GAPDH (ProteinTech Group, Inc.; cat. no. 60004-1-Ig; dilution, 1:5,000) was used as the loading control. Goat anti-rabbit IgG-HRP and goat anti-mouse IgG-HRP (OriGene Technologies, Inc.; cat. nos. TA130024 and TA130005; dilution, 1:5,000) secondary antibodies were used at room temperature for $1 \mathrm{~h}$ (20). The software used for densitometry was ImageJ version 1.46 (National Institutes of Health).

MTT assay. $\mathrm{H} 4$ and LN-229 cells $(2,000-3,000)$ were seeded in 96-well plates, and after $24 \mathrm{~h}$, the cells were stimulated with or without FGF or IGF1 for 30 min before TMZ treatment. Dimethyl sulfoxide was used to dissolve the purple formazan. MTT activity was measured in triplicate on days 1, 2, 3, 4 and 5. Proliferation curves were constructed by plotting the average of the triplicate values calculated by optical density measurements at a wavelength of $570 \mathrm{~nm}$ by a $96-$ well plate reader (23).

Statistical analysis. Differentially expressed genes were detected by unpaired Student's t-test using SPSS version 13.0 (SPSS, Inc., Chicago, IL, USA). Differential genes between primary high-grade gliomas and recurrent gliomas after chemoradiotherapy were identified by Student's t-test. Kaplan-Meier survival analysis was used to estimate survival distribution. The log-rank test was applied to assess the statistical significance between the stratified survival groups using GraphPad Prism version 4.0 statistical software (GraphPad Software, Inc.). KEGG pathway analysis was performed using DAVID (http://david.abcc.ncifcrf.gov/). Cut-off points for 
Table I. Established biomarkers in initial and recurrent high grade astrocytomas.

\begin{tabular}{|c|c|c|c|c|}
\hline \multirow[b]{2}{*}{ Variables } & \multicolumn{4}{|c|}{ Cases, $\%(\mathrm{n} / \mathrm{N})$} \\
\hline & Grade III & Grade IV & Recurrent Grade III & Recurrent Grade IV \\
\hline \multicolumn{5}{|l|}{ Sex } \\
\hline Male & $63.8(30 / 47)$ & $62.5(55 / 88)$ & $60.0(15 / 25)$ & $63.6(14 / 22)$ \\
\hline Female & $36.2(17 / 47)$ & $37.5(33 / 88)$ & $40.0(10 / 25)$ & $36.4(8 / 22)$ \\
\hline \multicolumn{5}{|l|}{ Age (years) } \\
\hline$\leq 42$ & $44.7(21 / 47)$ & $27.3(24 / 88)$ & $72.0(18 / 25)$ & $36.4(8 / 22)$ \\
\hline$>42$ & $55.3(26 / 47)$ & $72.7(64 / 88)$ & $32.0(7 / 25)$ & $63.6(14 / 22)$ \\
\hline \multicolumn{5}{|c|}{ Cancer Genome Atlas subtype } \\
\hline Neural & $19.1(9 / 47)$ & $14.8(13 / 88)$ & $24.0(6 / 25)$ & $9.1(2 / 22)$ \\
\hline Proneural & $38.3(18 / 47)$ & $8.0(7 / 88)$ & $48.0(12 / 25)$ & $22.7(5 / 22)$ \\
\hline Classical & $29.8(14 / 47)$ & $36.4(32 / 88)$ & $12.0(3 / 25)$ & $22.7(5 / 22)$ \\
\hline Mesenchymal & $12.8(6 / 47)$ & $40.9(36 / 88)$ & $16.0(4 / 25)$ & $45.5(10 / 22)$ \\
\hline \multicolumn{5}{|l|}{ IDH1/2 } \\
\hline Mutation & $40.9(18 / 44)$ & $11.7(9 / 77)$ & $70.8(17 / 24)$ & $31.8(7 / 22)$ \\
\hline Wild-type & $59.1(26 / 44)$ & $88.3(68 / 77)$ & $29.2(7 / 24)$ & $68.2(15 / 22)$ \\
\hline \multicolumn{5}{|l|}{ EGFRvIII } \\
\hline Mutation & $10.6(5 / 47)$ & $14.8(13 / 88)$ & $4.0(1 / 25)$ & $4.5(1 / 22)$ \\
\hline Wild-type & $89.4(42 / 47)$ & $85.2(75 / 88)$ & $96.0(24 / 25)$ & $95.5(21 / 22)$ \\
\hline \multicolumn{5}{|l|}{ TP53 } \\
\hline Mutation & $17.6(3 / 17)$ & $24.4(11 / 45)$ & $7.7(1 / 13)$ & $16.7(2 / 12)$ \\
\hline Wild-type & $82.4(14 / 17)$ & $75.4(34 / 45)$ & $92.3(12 / 13)$ & $83.3(10 / 12)$ \\
\hline \multicolumn{5}{|l|}{ PTEN } \\
\hline Mutation & $26.7(4 / 15)$ & $20.9(9 / 43)$ & $0(0 / 12)$ & $18.2(2 / 11)$ \\
\hline Wild-type & $73.3(11 / 15)$ & $79.1(34 / 43)$ & $100(12 / 12)$ & $81.8(9 / 11)$ \\
\hline \multicolumn{5}{|l|}{ MGMT } \\
\hline Methylated & $58.3(21 / 36)$ & $40.0(34 / 85)$ & $62.5(15 / 24)$ & $44.4(8 / 18)$ \\
\hline Unmethylated & $41.7(15 / 36)$ & $60.0(51 / 85)$ & $37.5(9 / 24)$ & $55.6(10 / 18)$ \\
\hline \multicolumn{5}{|l|}{ TERT } \\
\hline Mutation & $52.6(20 / 38)$ & $34.9(22 / 63)$ & $17.6(3 / 17)$ & $31.6(6 / 19)$ \\
\hline Wild-type & $47.4(18 / 38)$ & $65.1(41 / 63)$ & $82.4(14 / 17)$ & $68.4(13 / 19)$ \\
\hline
\end{tabular}

IDH1/2, isocitrate dehydrogenase 1/2, Cytosolic; EGFRvIII, epidermal growth factor receptor variant III; TP53, tumor protein P53; PTEN, phosphatase and tensin homolog; MGMT, $\mathrm{O}^{6}$-methylguanine-DNA methyltransferase; TERT, telomerase reverse transcriptase.

different variables were set according to the median value. A two-sided P-value $<0.05$ was regarded to indicate a statistically significant difference.

\section{Results}

Alterations in the distribution of established biomarkers. Numerous reliable molecular markers, such as TP53 and IDH1 mutations, are accepted as frequent alterations in glioma development (4-6). In the present study, whether these markers are altered in glioma following chemoradiotherapy was investigated. A total of 182 high-grade glioma samples (47 primary grade III, 88 primary GBM, 25 recurrent grade III and 22 recurrent GBM samples) were collected with information regarding PTEN mutation status, IDH1/2 status, TP53 mutation status, MGMT promoter methylation status, EGFRvIII mutation status, TERT mutation status and TCGA subtype, from the CGGA dataset and are presented in Table I. It was observed that tumor recurrence was more common in older patients. There was a decline in the EGFRvIII, TP53, PTEN and TERT mutation rates from primary high-grade gliomas to recurrent gliomas after chemoradiotherapy. However, the frequency of IDH1/2 mutations and MGMT promoter methylation was increased in gliomas following chemoradiotherapy, which may explain the insensitivity of recurrent gliomas to TMZ chemotherapy (Table I).

Dysregulation of RTK kinase family members in high-grade gliomas after chemoradiotherapy. RTKs play important roles in glioma development, and alterations of RTKs can influence the results of chemoradiotherapy. In the present study, the RNA sequencing information of 135 primary high-grade 
Table II. Significantly changed receptor tyrosine kinases between initial and recurrent gliomas.

\begin{tabular}{llccr}
\hline Gene & \multicolumn{1}{c}{ Full name } & Location & Fold-change & P-value \\
\hline IGF1R & Insulin-like growth factor 1 receptor & $15 \mathrm{q} 26.3$ & 2.0 & 0.01 \\
MST1R & Macrophage stimulating 1 receptor & $3 \mathrm{p} 21.3$ & 1.8 & 0.01 \\
FLT4 & Fms-related tyrosine kinase 4 & $5 \mathrm{q} 35.3$ & 1.5 & 0.02 \\
FLT3 & Fms-related tyrosine kinase 3 & $13 \mathrm{q} 12$ & 0.7 & 0.02 \\
FGFR1 & Fibroblast growth factor receptor 1 & 8p11.23-p11.22 & 1.4 & 0.05 \\
AATK & Apoptosis-associated tyrosine kinase & $17 \mathrm{q} 25.3$ & 1.4 & 0.05 \\
\hline
\end{tabular}

A

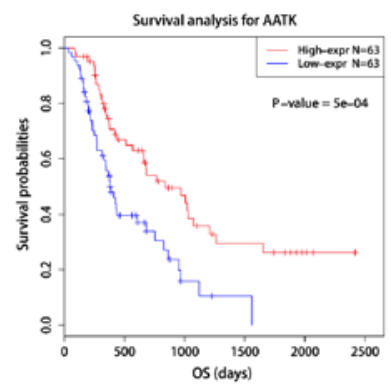

B
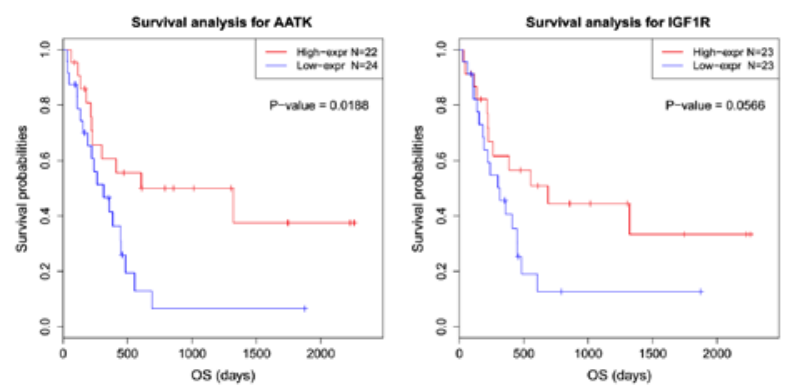

C
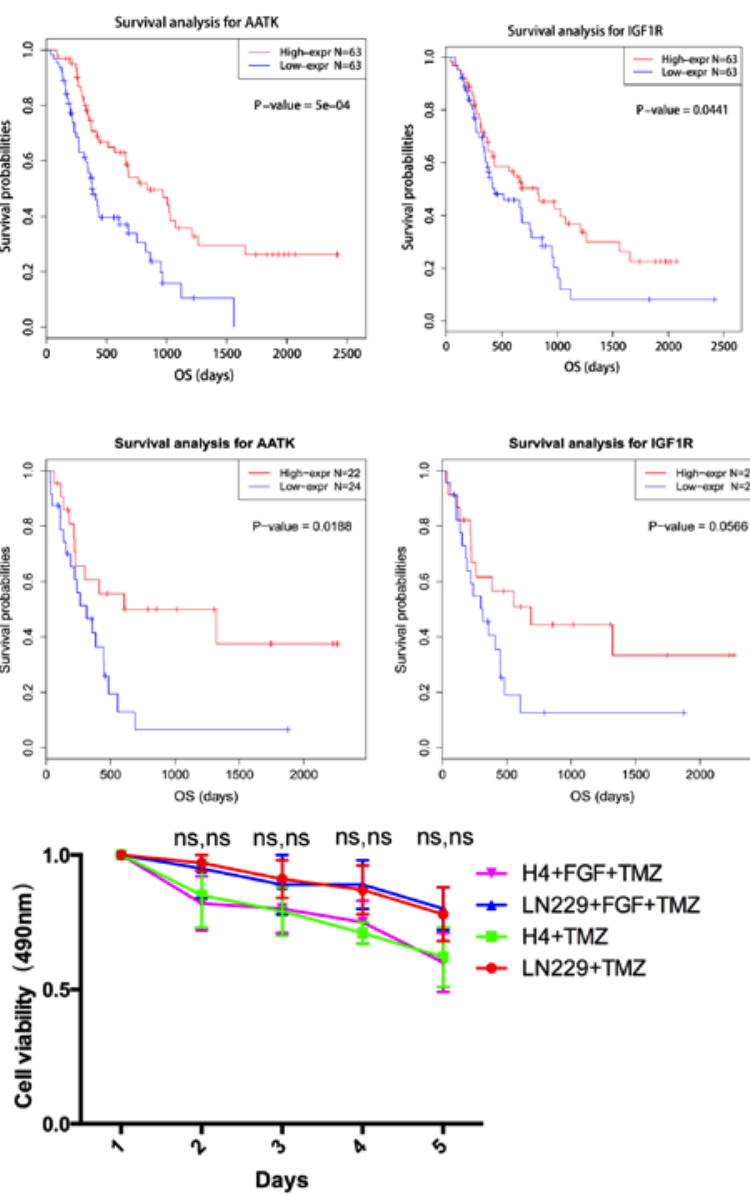
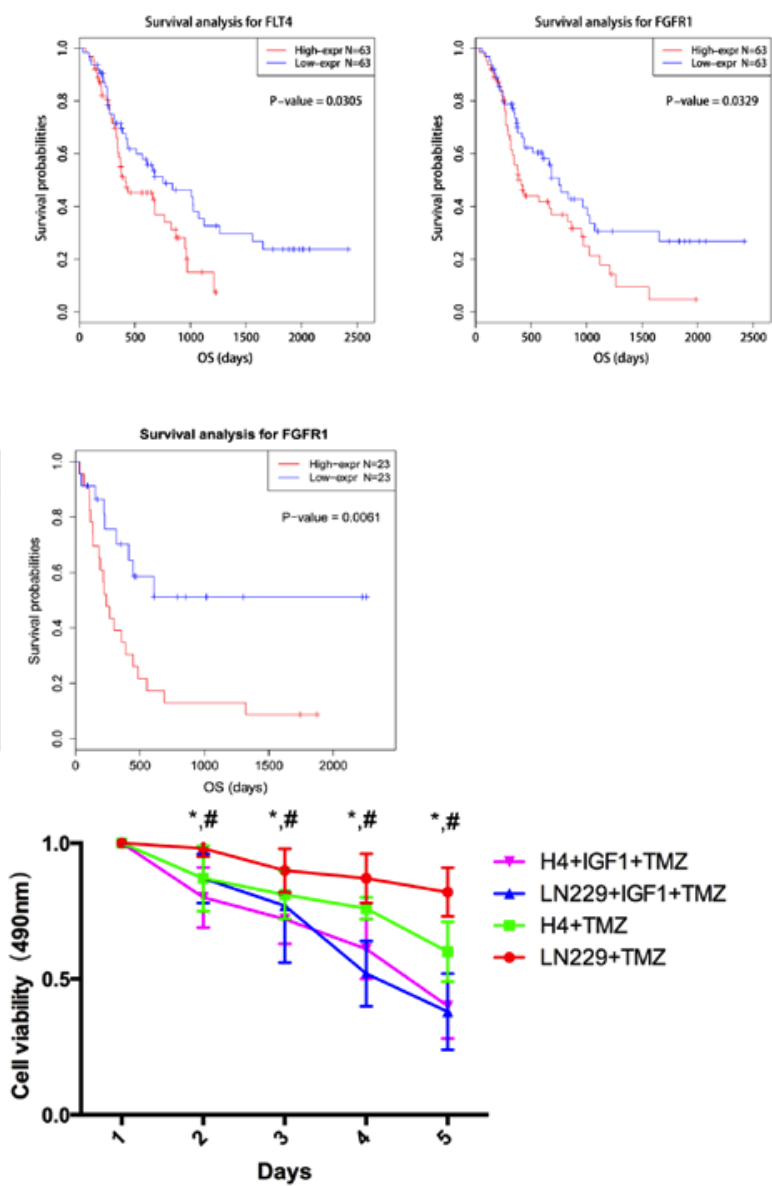

Figure 1. RTKs are associated with patient survival and cell viability. Expression levels of RTKs are associated with patient overall survival time in (A) initial and (B) recurrent high-grade glioma. (C) LN-229 and H4 glioma cells stimulated by IGF1 were sensitized to TMZ, whereas FGF did not sensitize the cells. The data are presented as mean \pm SD. ${ }^{*} \mathrm{P}<0.05$ for LN-229 + IGF1 + TMZ vs. LN229 + TMZ; ${ }^{\#} \mathrm{P}<0.05$ for H4 + IGF1 + TMZ vs. H4 + TMZ. RTK, receptor tyrosine kinase; AATK, apoptosis-associated tyrosine kinase; IGF1, insulin-like growth factor 1; FGF, fibroblast growth factor; FLT4, Fms-related tyrosine kinase 4; OS, overall survival; TMZ, temozolomide.

gliomas and 47 recurrent high-grade gliomas, following standardized radiotherapy and $\geq 1$ cycle of TMZ chemotherapy, was analyzed for the expression of 58 RTKs. The expression of 6 RTKs, including IGF1R, macrophage stimulating 1 receptor, Fms-related tyrosine kinase (FLT)4, FLT3, fibroblast growth factor receptor 1 (FGFR1) and apoptosis-associated tyrosine kinase (AATK), was demonstrated to be significantly altered after chemoradiotherapy. The expression of these RTKs, with the exception of FLT4, was increased in recurrent gliomas after chemotherapy (Table II).
RTKs are associated with prognosis and tumor cell viability. Among the collected samples, 126 primary high-grade glioma cases and 46 recurrent high-grade glioma cases had accessible survival information. Survival analysis indicated that high expression of IGF1R and AATK, and low expression of FLT4 and FGFR1 predicted longer overall survival time in primary glioma (Fig. 1A). In recurrent gliomas, high expression of AATK and IGF1R, and low expression of FGFR1 were associated with shorter overall survival time (Fig. 1B). Previous reports have indicated that the high 

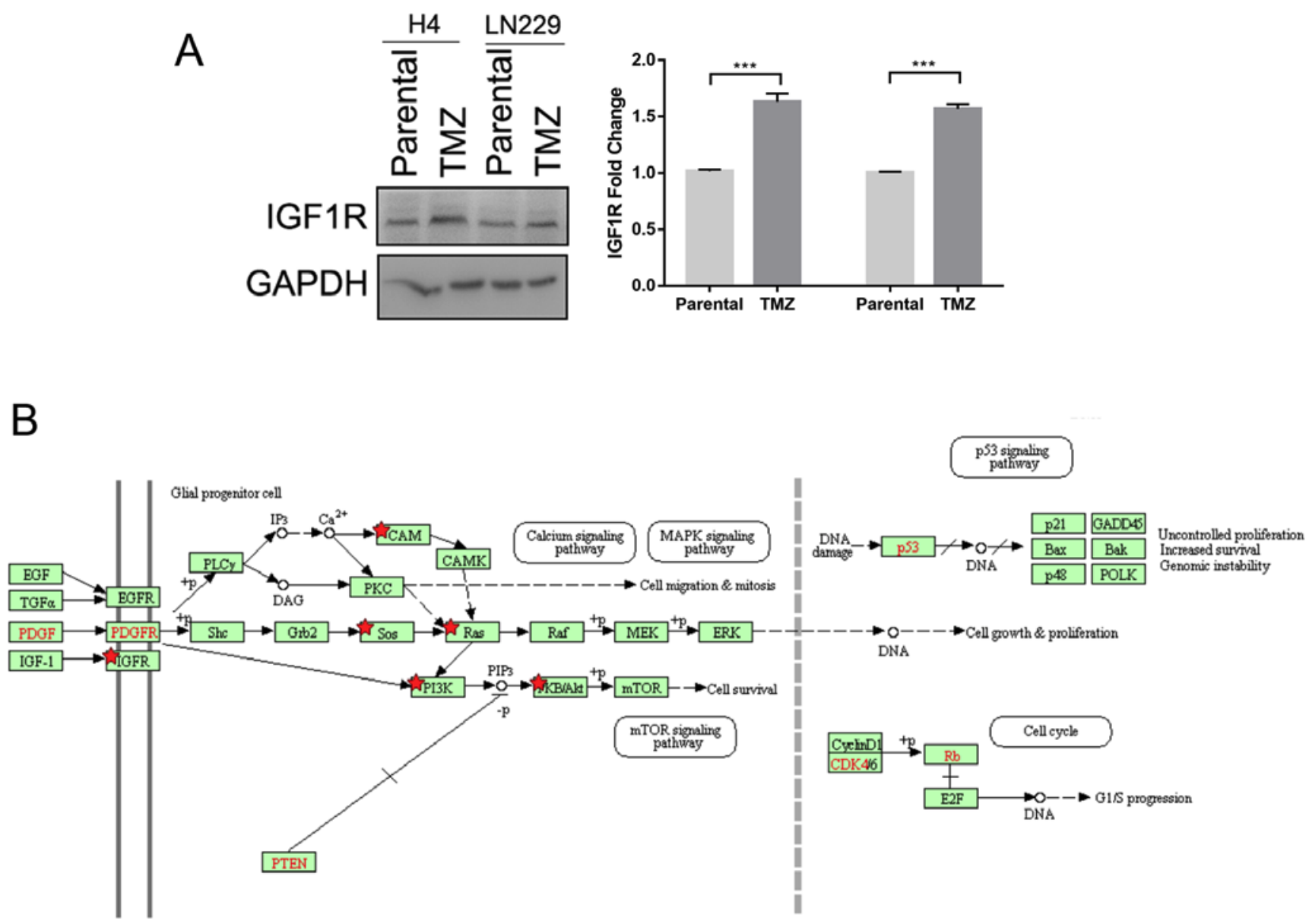

Figure 2. IGFR was activated by TMZ. (A) Western blot analysis revealed that IGF1R expression was increased in glioma cells after treatment with TMZ. Expression levels was normalized by the level of GAPDH and expressed as fold-changes of those in parental cells. The data are presented as mean \pm SD. ${ }_{* * * *} \mathrm{P}<0.001$ as indicated. (B) Data analysis of 135 initial and 47 recurrent high-grade glioma cases revealed that IGFR signaling was highly activated in recurrent gliomas. TMZ, temozolomide; IGF1R, insulin-like growth factor 1 receptor.

expression of IGF1R is an independent prognostic factor in tumors, including glioma and breast cancer (24-27). Clinical reports have also suggested that high activation of the Met (HGFR) signaling pathway by HGF can increase crizotinib-sensitivity in lung cancer $(22,24,25)$. Therefore, the RTK signaling pathway may influence treatment effectiveness. In the present study, FGF and IGF were used to stimulate tumor cells before TMZ treatment, and it was demonstrated that IGF stimulation assisted TMZ-induced tumor-cell death, whereas FGF did not induce sensitization to TMZ chemotherapy (Fig. 1C).

TMZ increases IGFIR expression and activates IGFIR signaling. RNA sequencing data revealed that the expression of IGF1R was increased in recurrent gliomas following treatment, indicative of activation of the IGF1R signal pathway (Table II). This was confirmed in the present study, in which the expression level of IGF1R was demonstrated to be increased in glioma cells after TMZ treatment (Fig. 2A). Differential genes between primary high-grade gliomas and recurrent gliomas after chemoradiotherapy were identified, and used to perform pathway analysis. The results indicated that the IGF1R signaling pathway was activated by TMZ treatment. The PDGFR signaling pathway was also demonstrated to be activated. These pathways regulate the cell cycle and promote cell growth, which may result in tumor-cell sensitivity to chemoradiotherapy (Fig. 2B).

\section{Discussion}

High-grade gliomas, particularly GBM, are the most common type of primary brain tumor, and have a high mortality rate (1). Surgical resection followed by adjuvant chemoradiotherapy is the standard mode of treatment; however, its effectiveness is limited (3). The biological effects of radiation are largely the result of DNA damage, which can induce cell death (16). However, cells attempt to repair the DNA injury induced by radiation via several pathways (16). Key genes affecting these radiation-repair pathways include ATM, which is associated with ataxia telangiectasia; $\mathrm{Ku}$, which is involved in the repair of double-strand DNA breaks; and XRCC2 (16). TMZ, an oral alkylating agent, is currently a first-line treatment for patients with high-grade glioma $(1,3)$. However, TMZ results in DNA methylation and the failure of MMR (12). A number of genes, including MGMT, can cause TMZ-resistance in cancer cells by repairing TMZ-induced DNA methylation (12). In the present study, the high frequency of IDH1/2 and MGMT promoter methylation in recurrent gliomas indicated that gliomas exhibiting these markers were more likely to recur or to be resistant to chemoradiotherapy. 
The RTK family regulates tumor development and treatment response and previous studies have found that alterations of RTK function can increase or reduce therapeutic resistance (13-15). A number of studies focused on IGF1R have identified that IGF1R expression is associated with numerous types of tumors, including glioma (26-29). IGF-1R has also been confirmed as a direct target gene of numerous microRNAs that are associated with glioma proliferation and invasion $(27,30)$. The IGF1R pathway has been reported to be associated with treatment resistance in glioma (29). In the present study, it was indicated that high expression levels of IGF1R increased sensitivity to chemoradiotherapy in high-grade gliomas. IGF1R serves a critical role in transformation events, particularly during epithelial-mesenchymal transition; it is highly overexpressed in malignant tissues and plays important roles in tumorigenesis, metastasis and resistance in numerous types of human tumors $(22,24,25)$. However, the prognostic relevance of IGF1R has long been a matter of controversy. High expression levels of IGF-1R have been associated with an increased risk of disease and poor survival rates in patients with tumors in certain studies, while other studies have demonstrated that high IGF-1R expression may predict a longer survival time and moderate sensitivity to anti-cancer drugs in other types of human tumors (25-28). Liang et al (24) reported that IGF-1 treatment affected the viability of classical Hodgkin lymphoma (cHL) cell lines, and increased the phosphorylation of IGF1R, Akt and ERK, while IGF-1R expression in Hodgkin and Reed-Sternberg cells predicted a favorable outcome, despite the oncogenic effect of IGF-1R in cHL cell lines. Mountzios et al (25) demonstrated that aberrant expression of components of the IGF1R pathway is associated with relatively good clinical outcomes in patients with luminal $\mathrm{A}$ and $\mathrm{B}$, node-positive early breast cancer, and suggested that hormone-receptor positive, HER2-negative tumors may explain, at least in part, the prognostic role of the IGFR pathway. Another large-sample study found that IGF1R mRNA expression was a prognostic marker in the entire cohort and in the luminal subtype groups $(22,24,25)$. In the present study, a high IGFR1 mRNA expression level was indicated to be also a prognostic factor for the survival of patients with high-grade glioma. Tumor cells stimulated by IGF1 recombinant cytokine exhibited increased sensitivity to TMZ treatment. Increased activation of IGF1R was also evident following radiotherapy and TMZ chemotherapy. IGF1R protein expression was increased in glioma cells treated with TMZ. These data suggest that IGF1R signaling may contribute to the response of tumor cells to chemoradiotherapy. The prognostic value of IGF1R may be dependent on its expression level in patients with gliomas.

A limitation of the present study is that although the use of paired tumor samples to compare the biological changes after treatment would have been the optimal approach, this was not possible. The reason is that during the course of treatment, the majority of patients either refused another surgery following recurrence, or underwent surgery elsewhere. Therefore, the collection of paired samples for analysis was limited. Further investigation of the downstream effectors of IGF1R is required. The differences in the expression of these genes in the dataset before and after chemoradiotherapy should be analyzed and the results further verified by cell experiments. In addition, the knockdown of key genes should be conducted to determine their effect on sensitivity to TMZ, as this may assist in selecting the optimal treatment for glioma.

In conclusion, the present study demonstrated that the expression levels of some RTKs are significantly altered after chemoradiotherapy in patients with glioma. These RTKs may serve important roles in the regulation of therapeutic sensitivity and the results of the present study may provide a basis for future research. High IGF-1R expression in patients with glioma predicts a favorable outcome, and may be included in future clinical risk stratification following validation by future large prospective studies.

\section{Acknowledgements}

Not applicable.

\section{Funding}

The present study was supported by funding from The National Nature Science Foundation of China (grant no. 81502495).

\section{Availability of data and materials}

The datasets generated and analyzed during the present study are available in the CGGA repository (http://www.cgga.org.cn).

\section{Authors' contributions}

$\mathrm{YL}$ and $\mathrm{KW}$ designed the experiments. $\mathrm{KW}, \mathrm{RH}, \mathrm{CW}$ and $\mathrm{ZZ}$ analyzed the data and contributed analytical tools. KW, GL and $\mathrm{HH}$ performed the experiments. $\mathrm{RH}$ and $\mathrm{KW}$ wrote the paper.

\section{Ethics approval and consent to participate}

This study was approved by the Ethics Committee of Beijing Tiantan Hospital (Beijing, China), and written informed consent was obtained from each patient.

\section{Patient consent for publication}

Not applicable.

\section{Competing interests}

The authors declare that they have no competing interests.

\section{References}

1. Jiang T, Mao Y, Ma W, Mao Q, You Y, Yang X, Jiang C, Kang C, Li X, Chen L, et al: CGCG clinical practice guidelines for the management of adult diffuse gliomas. Cancer Lett 375: 263-273, 2016.

2. Louis DN, Ohgaki H, Wiestler OD, Cavenee WK, Burger PC, Jouvet A, Scheithauer BW and Kleihues P: The 2007 WHO classification of tumours of the central nervous system. Acta Neuropathol 114: 97-109, 2007.

3. Stupp R, Brada M, van den Bent MJ,Tonn JC and Pentheroudakis G; ESMO Guidelines Working Group: High-grade glioma: ESMO clinical practice guidelines for diagnosis, treatment and follow-up. Ann Oncol 25 (Suppl): iii93-iii101, 2014. 
4. Cancer Genome Atlas Research Network, Brat DJ, Verhaak RG, Aldape KD, Yung WK, Salama SR, Cooper LA, Rheinbay E, Miller CR, Vitucci M, et al: Comprehensive, integrative genomic analysis of diffuse lower-grade gliomas. New Engl J Med 372: 2481-2498, 2015.

5. Mock A, Geisenberger C, Orlik C, Warta R, Schwager C, Jungk C, Dutruel C, Geiselhart L, Weichenhan D, Zucknick M, et al: LOC283731 promoter hypermethylation prognosticates survival after radiochemotherapy in IDH1 wild-type glioblastoma patients. Int J Cancer 139: 424-432, 2016.

6. Turcan S, Rohle D, Goenka A, Walsh LA, Fang F, Yilmaz E, Campos C, Fabius AW, Lu C, Ward PS, et al: IDH1 mutation is sufficient to establish the glioma hypermethylator phenotype. Nature 483: 479-483, 2012.

7. Cairncross G, Wang M, Shaw E, Jenkins R, Brachman D, Buckner J, Fink K, Souhami L, Laperriere N, Curran W and Mehta M: Phase III trial of chemoradiotherapy for anaplastic oligodendroglioma: Long-term results of RTOG 9402. J Clin Oncol 31: 337-343, 2013.

8. Intergroup Radiation Therapy Oncology Group Trial 1942, Cairncross G, Berkey B, Shaw E, Jenkins R, Scheithauer B, Brachman D, Buckner J, Fink K, Souhami L, et al: Phase III trial of chemotherapy plus radiotherapy compared with radiotherapy alone for pure and mixed anaplastic oligodendroglioma: Intergroup radiation therapy oncology group trial 9402. J Clin Oncol 24: 2707-2714, 2006.

9. Kaloshi G, Benouaich-Amiel A, Diakite F, Taillibert S, Lejeune J, Laigle-Donadey F, Renard MA, Iraqi W, Idbaih A, Paris S, et al: Temozolomide for low-grade gliomas: predictive impact of $1 p / 19 q$ loss on response and outcome. Neurology 68: 1831-1836, 2007

10. Bettegowda C, Agrawal N, Jiao Y, Sausen M, Wood LD, Hruban RH, Rodriguez FJ, Cahill DP, McLendon R, Riggins G, et al: Mutations in CIC and FUBP1 contribute to human oligodendroglioma. Science 333: 1453-1455, 2011.

11. Yip S, Butterfield YS, Morozova O, Chittaranjan S, Blough MD, An J, Birol I, Chesnelong C, Chiu R, Chuah E, et al: Concurrent CIC mutations, IDH mutations, and 1p/19q loss distinguish oligodendrogliomas from other cancers. J Pathol 226: 7-16, 2012.

12. Hegi ME, Diserens AC, Gorlia T, Hamou MF, de Tribolet N, Weller M, Kros JM, Hainfellner JA, Mason W, Mariani L, et al MGMT gene silencing and benefit from temozolomide in glioblastoma. N Engl J Med 352: 997-1003, 2005.

13. Schlessinger J: Cell signaling by receptor tyrosine kinases. Cell 103: 211-225, 2000

14. Regad T: Targeting RTK signaling pathways in cancer. Cancers(Basel) 7: 1758-1784, 2015.

15. Sundaram MV: RTK/Ras/MAPK signaling. WormBook: 1-19, 2006.

16. Blume-Jensen $P$ and Hunter T: Oncogenic kinase signalling. Nature 411: 355-365, 2001

17. Ou SH, Soo RA, Kubo A, Kawaguchi T and Ahn MJ: Will the requirement by the US FDA to simultaneously Co-develop companion diagnostics (CDx) delay the approval of receptor tyrosine kinase inhibitors for RTK-rearranged (ROS1-, RET-, AXL-, PDGFR- $\alpha-$, NTRK1-) non-small cell lung cancer globally? Front Oncol 4: 58, 2014

18. Hata AN, Niederst MJ, Archibald HL, Gomez-Caraballo M, Siddiqui FM, Mulvey HE, Maruvka YE, Ji F, Bhang HE, Krishnamurthy Radhakrishna V, et al: Tumor cells can follow distinct evolutionary paths to become resistant to epiderma growth factor receptor inhibition. Nat Med 22: 262-269, 2016.
19. Xie Q, Bradley R, Kang L, Koeman J, Ascierto ML, Worschech A, De Giorgi V, Wang E, Kefene L, Su Y, et al: Hepatocyte growth factor (HGF) autocrine activation predicts sensitivity to MET inhibition in glioblastoma. Proc Natl Acad Sci USA 109: 570-575, 2016.

20. Liu Y, Hu H, Wang K, Zhang C, Wang Y, Yao K, Yang P, Han L, Kang C, Zhang W and Jiang T: Multidimensional analysis of gene expression reveals TGFB1I1-induced EMT contributes to malignant progression of astrocytomas. Oncotarget 5: 12593-12606, 2014.

21. Cartland SP, Genner SW, Zahoor A and Kavurma MM: Comparative evaluation of TRAIL, FGF-2 and VEGF-A-induced angiogenesis in vitro and in vivo. Int J Mol Sci 17: pii: E2025, 2016.

22. Gong Y, Yao E, Shen R, Goel A, Arcila M, Teruya-Feldstein J, Zakowski MF, Frankel S, Peifer M, Thomas RK, et al: High expression levels of total IGF-1R and sensitivity of NSCLC cells in vitro to an anti-IGF-1R antibody (R1507). PLoS One 4: e7273, 2009.

23. Zhou Y, Li S, Li J, Wang D and Li Q: Effect of microRNA-135a on cell proliferation, migration, invasion, apoptosis and tumor angiogenesis through the IGF-1/PI3K/Akt signaling pathway in non-small cell lung cancer. Cell Physiol Biochem 42: 1431-1446, 2017.

24. Liang Z, Diepstra A, Xu C, van Imhoff G, Plattel W, Van Den Berg A and Visser L: Insulin-like growth factor 1 receptor is a prognostic factor in classical Hodgkin lymphoma. PLoS One 9: e87474, 2014.

25. Mountzios G, Aivazi D, Kostopoulos I, Kourea HP, Kouvatseas G, Timotheadou E, Zebekakis P, Efstratiou I, Gogas H, Vamvouka C, et al: Differential expression of the insulin-like growth factor receptor among early breast cancer subtypes. PLoS One 9: e91407, 2014.

26. Almiron Bonnin DA, Ran C, Havrda MC, Liu H, Hitoshi Y, Zhang Z, Cheng C, Ung M and Israel MA: Insulin-mediated signaling facilitates resistance to PDGFR inhibition in proneural hPDGFB-driven gliomas. Mol Cancer Ther 16: 705-716, 2017.

27. Wang H, Tang C, Na M, Ma W, Jiang Z, Gu Y, Ma G, Ge H, Shen $\mathrm{H}$ and Lin Z: miR-422a inhibits glioma proliferation and invasion by targeting IGF1 and IGF1R. Oncol Res 25: 187-194, 2017.

28. Arun S, Vanisree AJ and Ravisankar S: Connexin 30 downregulates Insulin-like growth factor receptor-1, abolishes Erk and potentiates effects of an IGF-R inhibitor in a glioma cell line. Brain Res 1643: 80-90, 2016.

29. Ma Y, Tang N, Thompson RC, Mobley BC, Clark SW, Sarkaria JN and Wang J: InsR/IGF1R pathway mediates resistance to EGFR inhibitors in glioblastoma. Clin Cancer Res 22: 1767-1776, 2016.

30. Jiang J, Wang W, Fang D, Jin X, Ding L and Sun X: MicroRNA-186 targets IGF-1R and exerts tumor-suppressing functions in glioma. Mol Med Rep 16: 7821-7828, 2017.

This work is licensed under a Creative Commons Attribution-NonCommercial-NoDerivatives 4.0 International (CC BY-NC-ND 4.0) License. 